\title{
EXTRAORAL TOOTH 48 EXTRACTION - A SEQUENCE OF MEDICAL ERRORS AND COMPLICATIONS
}

\author{
Anna Smędra' ${ }^{1}$ Katarzyna Wochna', Aneta Neskoromna-Jędrzejczak², Jarosław Berent ${ }^{1}$ \\ ${ }^{1}$ Chair and Department of Forensic Medicine, Medical University of Lodz, Poland \\ ${ }^{2}$ Craniomaxillofacial Surgery Clinic, $2^{\text {nd }}$ Chair of Surgery, Medical University of Lodz, Poland
}

\begin{abstract}
INTRODUCTION: Extraction of unerupted wisdom teeth may be associated with a number of potential complications such as postoperative bleeding, infection, dry socket, neurological disorders, intraoperative fracture of the mandible, osteitis, temporary or permanent numbness (paresthesia), abscesses, and hematomas. The literature also contains reports concerning late fractures of the mandible that are complicated with osteitis and required repair with autogenic transplantation. In most cases, the classical intra-oral approach for dental extraction dominates because the extraoral approach is connected with more potential complications, specifically nerve and vascular damage, joint injuries and skin scarring, as a result of surgery.

CASE REPORT: This paper presents a case of irregularities and complications that occurred during and after the treatment of a female patient who required extraction of tooth 48 (the right lower third molar). First, a procedure other than the treatment that had been planned was performed (removal of a cyst from the right maxillary sinus); then, the second procedure (extraction of tooth 48) was performed without the patient's consent and with inappropriate extraoral access. Ultimately, the complication that occurred during the treatment, i.e., a fracture of the mandible, was not recognized. The patient was treated for months by various physicians, and eventually, after 8 months, an inveterate fracture of the mandible, requiring reconstructive treatment, was diagnosed. The patient reported the case to the Public Prosecutor's Office, which ordered an opinion to determine whether the implemented medical procedure was correct.

Conclusions: The described case shows that sometimes the course of treatment can lead to a seemingly incredible accumulation of irregularities and complications. The forensic medical opinion allowed the indictment of the doctor responsible for the irregularities. As a result of the mediation proceedings, the parties managed to avoid a long and costly process and the patient received compensation and reparation.
\end{abstract}

KEY WORDS: maxillo-facial surgery, medical error, complication, forensic medical opinion, mediation, compensation.

J Stoma 2018; 71, 5: 439-443

DOI: https://doi.org/10.5114/jos.2018.84646

\section{INTRODUCTION}

The third molars, colloquially referred to as wisdom teeth, erupt most frequently between 17 and 21 years of age but sometimes considerably later or even not at all.
The wisdom teeth do not always erupt completely, they can sometimes grow at a wrong angle, and they are the most susceptible of all teeth to caries, as a result of both difficult toothbrush access and food residues remaining in their vicinity. For this reason, and because they are barely in-

\section{JOURNAL OF} STOMATOLOGY CZASOPISMO STOMATOLOGICZNE

AdDress For Correspondence: Anna Smędra, Chair and Department of Forensic Medicine, Medical University of Lodz, 18a Sędziowska St., 91-304 Łódź, Poland, phone: +48 50825 76 57, fax: +48 4265442 93, 
volved in chewing, until recently, it was believed that these teeth should always be extracted because they are useless and associated with numerous potential problems. Currently, they are extracted most commonly as a result of medical conditions that fail to respond to conservative treatment, as well as for orthodontic and prosthetic reasons.

In a situation when extraction of an unerupted wisdom tooth is necessary, the transoral approach is used, i.e., from the occlusal surface or from the vestibular surface of the retained tooth. If both approaches fail to expose the unerupted tooth with its subsequent extraction as needed, extraoral access is the last resort as it is associated with significant destruction of the bone in the surgical area. Extraction of unerupted wisdom teeth may be associated with a number of potential complications such as postoperative bleeding, infection, dry socket, neurological disorders (e.g., of the inferior alveolar nerve or lingual nerve), or intraoperative fracture of the mandible $[1,2]$. Osteitis, temporary or permanent numbness (paresthesia), abscesses, and hematomas have also been reported [3].

The literature also contains reports concerning late fractures of the mandible (invisible on the radiological images obtained immediately after the extraction) that are complicated with osteitis and required repair with autogenic transplantation [4]. Krimmel et al. described the case of a mandibular fracture that occurred 14 days after the extraction of an unerupted tooth; the authors described the attempt to determine the predisposing factors for this complication [5]. Libersa et al. discussed intraoperative and late fractures of the mandible [6]. Additionally, some cases of abscesses that developed in the pterygomandibular space [7] or in the brain [8], requiring neurosurgery and antibiotic treatment, have been described. There are also descriptions of the displacement of unerupted wisdom teeth to the submandibular space [9] and the sublingual space [10]. In most cases, the classical intra-oral approach for dental extraction dominates because the extraoral approach is connected with more potential complications, specifically nerve and vascular damage, joint injuries and skin scarring, as a result of surgery [11]. However, when intra-oral surgery is impossible, extraoral approaches might be useful, especially for the removal of ectopic third molars or in cases of severe trismus [12].

The literature highlights the key role of the operator's experience in reducing the incidence of post-extraction complications [2]. Sometimes, when complications occur in the course of treatment, it ends in preparatory proceedings to determine whether this incident was included in the risk of the therapy or was the result of misconduct and was a de facto medical error. In such situations, the case is referred to experts to prepare a medical opinion about the correctness of the medical treatment.

The aim of this paper was to discuss an unusual case that had a number of irregularities and complications that occurred during the treatment of a female patient who required extraction of tooth 48 , assessed with respect to the correctness of the medical procedures and the effect of the forensic medical opinion concerning the case in further court proceedings and mediation. The authors obtained court permission to publish this case.

\section{CASE REPORT}

On April 3, 2013, a 34-year-old woman sought assistance in a dental clinic because of painful symptoms as a result of the eruption of retained tooth 48 (the right lower third molar). On that date, the tooth could not be removed in the dental office.

The patient was referred to a maxillofacial surgery department. She was advised to obtain a radiograph of tooth 48 (Figure 1). In addition, she received a prescription for an antibiotic, Dalacin C $300 \mathrm{mg}$ (clindamycin), 1 package.

The next day, the patient was seen by a maxillary surgeon. After yet another failed attempt to extract the tooth in the outpatient department, the patient qualified for surgical removal of the tooth under general anesthesia.

On April 12, 2013, she was admitted to the maxillofacial surgery department. On admission, it was noted that an earlier X-ray revealed the presence of a cyst in the right maxillary sinus in addition to retained tooth 48 .

The medical records contained the patient's consent to the right maxillary sinus cystectomy that was to be performed under general anesthesia; there was no consent regarding retained tooth 48 extraction surgery from the extraoral incision approach under general anesthesia. As follows from the patient's testimony, she thought she signed consent for tooth 48 extraction.

According to the patient's testimony, on the day of admission, she underwent general anesthesia twice and two surgical procedures were performed. After waking after the first treatment involving removal of the cyst from the right maxillary sinus, she noticed that her retained tooth 48 had not been removed. She was transferred again to the operating room, where she underwent a second administration of general anesthesia and an operation to remove the retained tooth from the extraoral approach (there are no anesthesiological records confirming the second medical procedure). The medical records did not contain a precise description of the performed procedures. The results of the histopathological investigation of the resected maxillary sinus cyst revealed signs of a chronic inflammatory condition.

The postoperative condition of the patient was evaluated to be good; she was discharged the next day, i.e., April 13, 2013, with the recommendations of a less active lifestyle and avoidance of injuries to the mandible.

Almost two weeks after the treatment, the patient came to the operator to remove the dressing. During this visit, she reported a lack of sensation on the right side of her face. The doctor said that the feeling would come back after a few months. 
After that visit, the patient was seen by a primary care physician, who diagnosed her with right-sided paralysis of nerve VII, i.e., falling of the right corner of the mouth and paresthesias in the right cheek. The patient was treated with a steroid, Metypred (methylprednisolone).

In May and June 2013, she underwent physiotherapy of the facial nerve (electrical stimulation and galvanization).

At the end of June 2013, the patient sought assistance in the neurological clinic. Slight asymmetry of the mouth on the right side was found. Nivalin (galantamine), a drug used for damage to peripheral nerves and impaired conduction of nerve impulses, was instituted. The patient was seen at the clinic twice more. No improvement was observed after the treatment.

From June 2013, the patient also remained under the care of the ear, nose and throat (ENT) clinic. She complained of tingling and an asymmetrical position of the mouth while whistling, as well as abnormal sensations in the area of the right cheek. The doctor described the presence of a scar in the mandibular region on the right side.

At the end of August 2013, during the next visit to the ENT clinic, a hard lump in the right side of the submandibular salivary gland was described; a referral to an inpatient ENT department was issued, where she was diagnosed with inflammation of the right submandibular salivary gland.

At the beginning of December 2013, a panoramic $\mathrm{X}$-ray of the mandible was taken. It revealed an oval osteolytic change around the right mandibular angle with dimensions of $35 \times 22 \mathrm{~mm}$; within this osteolytic change, a fragment of the mandibular ramus with a sequestrum was visible. Urgent consultation in the maxillofacial surgery department was recommended (Figure 2).

In mid-January 2014, the patient was seen at the hospital and qualified for surgery because of an inveterate fracture of the mandible.

In February and March 2014, she stayed in an inpatient maxillofacial surgery department for treatment of the existing mandibular fracture. It was noted that the fracture had not been previously identified or treated; as a result, pseudoarthrosis developed. Based on the anamnesis, it was found that a few months after the surgery, there was a breakthrough of the bone through the skin in the region of the right mandibular angle. Shortening of the right mandibular body was found clinically and radiologically, and the jaw was reconstructed using an autogenic iliac bone graft and a RECO fixing plate. The surgery was performed under general anesthesia from an extraoral approach. The postoperative course was uneventful. Elastic intermaxillary fixation was maintained throughout. On the $7^{\text {th }}$ day, the sutures were removed and the patient was discharged home with recommendations, among others, to maintain the elastic fixation for 3 weeks.

The aggrieved person asked for judgment against the defendant including an obligation to repair the damage, as well as compensation for the harm suffered with

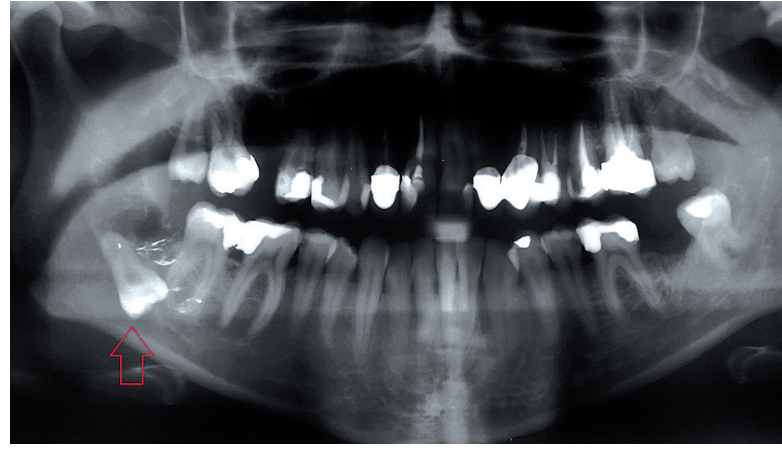

FIGURE 1. Preoperative X-ray of retained tooth 48 from 4 April 2013; the radiograph shows the location of retained tooth 48 . The radiopaque space above the root of tooth 48 is bone loss caused by chiseling the tooth the day before (during the unsuccessful attempt to extract the tooth in the outpatient department)

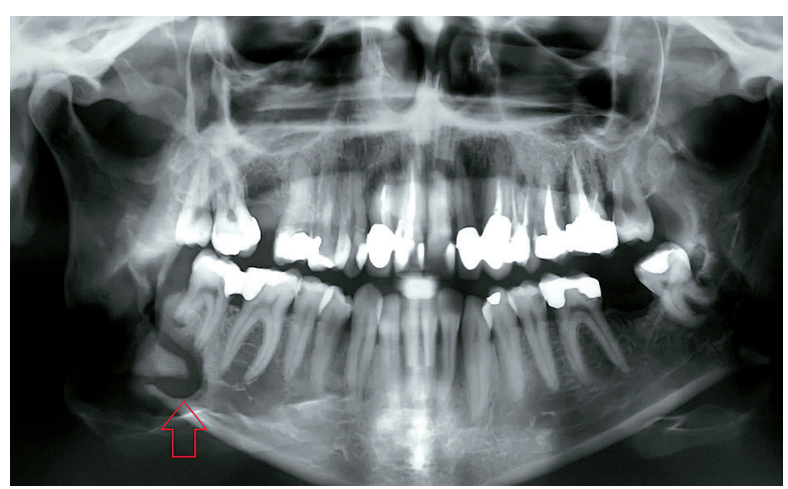

FIGURE 2. Postoperative X-ray from 5 December 2013; arrow shows a pathological fracture of the body of the mandible

a payment of 120 thousand zlotys (equivalent to approx. 33 thousand dollars, 28 thousand euros). The case was referred to experts.

The experts found that the surgery to remove the right maxillary sinus cyst, of non-dental origin, which in fact did not cause any pain, did not have to be performed at that time; the patient only wanted retained tooth 48 to be extracted. In addition, the Caldwell-Luc approach selected by the operator for the treatment is a destructive method that is radical in its premise. In view of the fact that the cyst was non-odontogenic, the patient should have qualified for sparing treatment by direct endoscopy, which does not entail the risk of complications in the form of hypoesthesia spectrum paresthesias of the right side of the face. Therefore, qualification of the patient for Caldwell-Luc surgery was considered inappropriate.

Similarly, qualification of the patient for surgery to remove retained tooth 48 via an extraoral approach was incorrect management with no substantive justification. There were no indications for the necessity of using such an approach based on the position of tooth 48 that was 


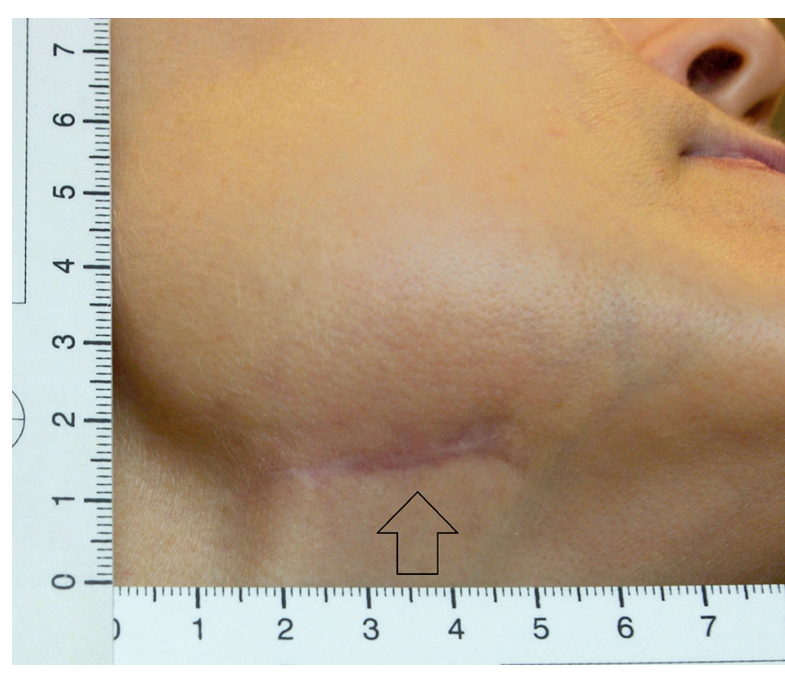

FIGURE 3. Scar in the right submandibular region from 26 November 2014; a photograph taken during the forensic medical examination after completion of the treatment

visible on the panoramic radiograph; moreover, there were no skin fistulas associated with chronic inflammation in the region of tooth 48 . Additionally, the continuity of the lower edge of the mandibular body in the vicinity of retained tooth 48 also pointed to the merits of its extraction via intraoral access.

Surgical removal of a retained tooth carries the risk of an iatrogenic mandibular fracture of a tooth. Such a complication is included in the risk of the surgery and may occur even if the procedure is performed correctly. Therefore, after the surgery, the mandible should be palpated to check whether its continuity has been maintained and there is no pathologic mobility of bone fragments. In the case of detecting mandibular fractures, with the patient still under general anesthesia, it is possible to fix fractures of the mandible immediately, during the same procedure.

Based on the analysis of the case files, it was impossible to establish whether the mandibular fracture occurred at the time of the surgery, or later, during the hospitalization, or after the patient's discharge from the hospital. Taking into account the testimony of the aggrieved person, according to which she had been informed that "the lower jaw was incised twice, at the top and at the bottom and it is held together on a delicate little bone", as well as the information included in the recommendations section of the discharge summary that she had to avoid trauma to the mandible, it can be presumed that the fracture did not occur during the surgery. However, because the operator did not order a control panoramic X-ray after the surgery, this could not be determined unequivocally. It should be added that, after extensive surgery, diagnostic imaging should be carried out to exclude a possible fracture of the mandible.

The applied surgical technique, involving incision of the mandible from the top and from the bottom, was incomprehensible, but the application of such a technique increased the possibility of a fracture of the lower jaw; hence, appropriate diagnostics and treatment should have been implemented. In the case of a mandibular fracture, the patient would have required stabilization of the bone fragments and osteosynthesis with titanium plates, or, in the case of the non-availability of surgical treatment, temporary orthopedic immobilization and referral for further management to a higher level reference center for osteosynthetic treatment would be implemented. If the procedure resulted in weakening of the bone structure, orthodontic treatment consisting of the use of dental splints and intermaxillary traction should have been instituted.

In summary, the following irregularities were found: 1. The original cause of the patient seeking treatment in the hospital ward in April 2013, according to medical records and the testimony of the victim, was, in fact, the retention of tooth 48 . Therefore, at that time there was no need for the surgery to remove the right maxillary sinus cyst. Only the procedure to remove tooth 48 should have been performed then, via intraoral access.

2. Apart from the fact that in April 2013 there was a need for surgery to remove a cyst in the right maxillary sinus, the procedure - if performed - should have been accomplished by sparing direct endoscopy and not by the Caldwell-Luc method. The latter method resulted in hypoesthesia of the right half of the patient's face, which would not have occurred with the sparing method.

3. No informed consent to surgery under general anesthesia to remove the tooth was obtained, and the patient was not informed about potential complications related to the surgery.

4. The patient received no preventive antibiotic either before or after the surgery in the hospital.

5. In the course of the procedure, the bone structure was weakened (the mandible was incised both from the bottom and from the top), requiring the implementation of orthodontic treatment involving the use of dental splints and intermaxillary traction; these were not performed. This led to an iatrogenic fracture of the mandible, chronic inflammation of the mandibular body with the formation of the pseudoarthrosis, the presence of sequestra, a loss of compact bone around the fractures and a reduction of the length of the mandibular body. A consequence of the above was the need to perform further operations, i.e., reconstruction of the bone loss with an iliac bone graft, removal of tooth 47 from the fracture space, and stabilization of the bone fragments with a RECO reconstruction plate.

6. The data from the case file and the forensic medical examinations did not give reasons to assume that the committed irregularities, and in particular, the incorrect choice of treatment of retained tooth 48 via an extraoral instead of an intraoral ap- 
proach, as well as the failure to diagnose the mandibular fracture with all the consequences, including the need for extensive and burdensome repair treatment, resulted in exposure of the victim to direct danger of loss of life or severe detriment to her health. There was also no reason to assume that they led to any form of grievous bodily harm and, in particular, to sustainable, significant disfigurement within the meaning of the provisions of the criminal code. It could only be assumed that the patient suffered from an impairment of function of an organ, i.e., the masticatory system, lasting more than seven days, where it should be emphasized that the impairment period exceeded the criterion of seven days very significantly.

Based on the received opinion, an indictment was brought against the doctor. The court decided to refer the matter to the mediator for mediation proceedings. The mediation ended in a settlement. The doctor pleaded guilty, apologized to the aggrieved person and agreed to pay the agreed sum of money. The victim accepted the apologies of the defendant, declaring that she did not insist on his punishment, she only wanted to obtain the financial resources with which she would be able to undertake further treatment and that would provide financial compensation for the suffered harm.

The accused paid the amount of PLN 3500 (approx. 960 dollars, approx. 800 euros) as personal symbolic reparation for the damage. In terms of further claims for damages and civilian legal remedies, the victim had to contact the defendant's insurer. Under such circumstances, the court decided to terminate the criminal proceedings based on the aggrieved person's statement concerning reconciliation with the defendant.

\section{CONCLUSIONS}

The discussed case shows that sometimes the course of treatment can lead to a seemingly incredible accumulation of irregularities and complications. The forensic medical opinion issued in this case allowed the indictment of the doctor responsible for the irregularities and the referral of the case to the court. As a result of the mediation proceedings, the parties managed to avoid a long and costly process and the patient received compensation and reparation.

\section{CONFLICT OF INTEREST}

The authors declare no potential conflicts of interest with respect to the research, authorship, and/or publication of this article.

\section{References}

1. Pitekova L, Satko I, Novotnakova D. Complications after third molar surgery. Bratisl Lek Listy 2010; 111: 296-298.
2. Blondeau F, Daniel NG. Extraction of impacted mandibular third molars: postoperative complications and their risk factors. J Can Dent Assoc 2007; 73: 325

3. Sigron GR, Pourmand PP, Mache B, et al. The most common complications after wisdom-tooth removal: Part 1: A retrospective study of 1,199 cases in the mandible. Swiss Dental Journal 2014; 124: $1042-1056$

4. Wagner KW, Schoen R, Wongchuensoontorn C, Schmelzeisen R. Complicated late mandibular fracture following third molar removal. Quintessence Int 2007; 38: 63-65

5. Krimmel M, Reinert S. Mandibular fractures after third molar removal. J Oral Maxillofac Surg 2000; 58: 1110-1112.

6. Libersa P, Roze D, Cachart T, Libersa JC. Immediate and late fractures after third molar removal. J Oral Maxillofac Surg 2002; 60: 163-165.

7. Ramchandani PL, Sabesan T, Peters WJ. Subdural empyema and herpes zoster syndrome (Hunt syndrome) complicating removal of third molars. Br J Oral Maxillofac Surg 2004; 42: 371.

8. Revol P, Gleizal A, Kraft T, et al. Brain abscess and diffuse cervico-facial cellulites: complication after third molar extraction. Rev Stomatol Chir Maxillofac 2003; 104: 285-289.

9. Yalcin S, Aktas I, Emes Y, Atalay B. Accidental displacement of a high-speed handpiece bur during mandibular third molar surgery: a case report. Oral Surg Oral Med Oral Pathol Oral Radiol Endod 2007; 105: 29-31.

10. Koseoglu BG, Gumru O, Kocaelli HA. Lower third molar displaced in the sublingual space. Dentomaxillofacial Radiology 2002; 31: 393.

11. Makhoul R, Delbet-Dupas C, Even J, et al. Atypical surgical approaches for removal of ectopic third molars: report of three cases. Med Buccale Chir Buccale 2016; 22: 233-238.

12. Christensen RO. Extraoral removal of an impacted lower third molar: report of case. J Oral Surg (Chic) 1950; 8: 335-337. 